\title{
Tailoring light polarization in vertical cavity surface emitting lasers by isotropic optical feedback from an extremely short external cavity
}

Cite as: Appl. Phys. Lett. 89, 091102 (2006); https://doi.org/10.1063/1.2339040

Submitted: 19 May 2006 . Accepted: 07 July 2006 . Published Online: 28 August 2006

Mikel Arizaleta Arteaga, Manuel López-Amo, Hugo Thienpont, and Krassimir Panajotov

\section{ARTICLES YOU MAY BE INTERESTED IN}

Polarized optical feedback from an extremely short external cavity for controlling and stabilizing the polarization of vertical cavity surface emitting lasers

Applied Physics Letters 90, 121104 (2007); https://doi.org/10.1063/1.2714301

Role of external cavity reflectivity for achieving polarization control and stabilization of vertical cavity surface emitting laser

Applied Physics Letters 90, 031117 (2007); https://doi.org/10.1063/1.2431790

This article may be downloaded for personal use only. Any other use requires prior permission of the author and AIP Publishing

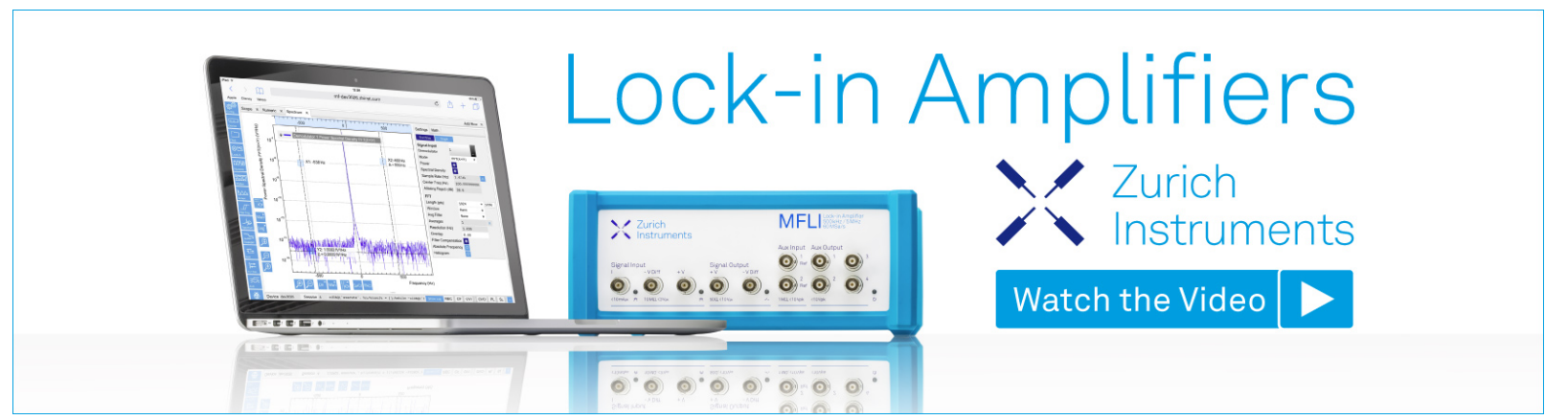




\title{
Tailoring light polarization in vertical cavity surface emitting lasers by isotropic optical feedback from an extremely short external cavity
}

\author{
Mikel Arizaleta Arteaga ${ }^{a)}$ and Manuel López-Amo \\ Department of Electrical and Electronic Engineering, Universidad Pública de Navarra, \\ Campus Arrosadía, 31006 Pamplona, Navarra, Spain \\ Hugo Thienpont and Krassimir Panajotov ${ }^{\text {b) }}$ \\ Department of Applied Physics and Photonics, Vrije Universiteit Brussel, Pleinlaan 2, 1050 Brussels, \\ Belgium
}

(Received 19 May 2006; accepted 7 July 2006; published online 28 August 2006)

\begin{abstract}
The authors experimentally show that isotropic optical feedback from an extremely short external cavity can induce switching between the two fundamental transverse modes with orthogonal linear polarization of otherwise (without optical feedback) polarization stable vertical cavity surface emitting laser. The switching currents and the hysteresis width can be widely tuned by varying the external cavity length. Moreover, by a proper choice of the optical feedback parameters, the emission can be stabilized in any of the two linearly polarized modes. (C) 2006 American Institute of Physics. [DOI: 10.1063/1.2339040]
\end{abstract}

Due to their high performance and low cost vertical cavity surface emitting lasers (VCSELs) have become very strong competitors to edge emitting lasers. VCSELs, however, suffer from a nonstable polarization behavior which might prevent their implementation in some applications, e.g., optical sensing and laser absorption spectroscopy. The cylindrical symmetry of the cavity, the polarization independent mirrors, and the lasing direction, perpendicular to the surface of the quantum wells, make VCSELs have no a priori defined polarization direction. In practice, small anisotropies introduced in the manufacturing process define two preferred polarization directions of the fundamental transverse mode along the [011] and [0-11] crystal axes in VCSELs grown on [100] oriented substrate. ${ }^{1,2}$ These two orthogonally linearly polarized (LP) modes have slightly different frequencies of operation and net gains, and polarization switching (PS) between them may occur when changing the injection current. ${ }^{3,4}$ Many different techniques aimed to introduce polarization dependent modal gain and/or losses have been proposed to avoid the switching and fix the polarization state of the emitted light from fabrication, e.g., etching of rectangular mesas ${ }^{5}$ or surface grating, ${ }^{6}$ growth on misoriented substrates, ${ }^{7}$ etc. Isotropic and polarized optical feedback from short and long external cavities ${ }^{8-13}$ and optical injection ${ }^{14}$ have also been proposed to achieve polarization stabilization or polarization control. Taking advantage of the practicably inevitable frequency difference between LP modes and using a mode selection mechanism similar to the one presented in Ref. 15, isotropic and polarized optical feedback from an extremely short external cavity (ESEC) has been proposed as a way to build a device whose polarization is actively controllable and stable against injection current variations. ${ }^{16}$ Here we present experimental evidences showing that active polarization control and polarization stabilization are indeed possible in VCSELs by means of isotropic optical feedback from an ESEC.

\footnotetext{
${ }^{a)}$ Electronic mail: mikel.arizaleta@unavarra.es

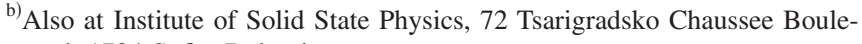
vard, 1784 Sofia, Bulgaria.
}

In the experiments we use an oxide-confined GaAs/InGaAs quantum well VCSEL, emitting at $1.005 \mu \mathrm{m}$ and supplied by the University of Ulm. The VCSEL is made with a circular oxide aperture and shallow surface relief in order to enhance the current region of single transverse mode operation. The VCSEL is emitting in its fundamental transverse mode up to 1.75 times the threshold current [see Fig. 1(a)], which has been confirmed from the optical spectrum measured with a high resolution $(0.01 \mathrm{~nm})$ Ando AQ6317 optical spectrum analyzer. All the experiments reported here are performed at a controlled substrate temperature of $26^{\circ} \mathrm{C}$. Figure 1(b) shows the experimentally obtained polarization resolved power versus current $(P I)$ curve of the solitary device, i.e., without optical feedback. The two represented modes correspond to the two fundamental transverse modes with orthogonal polarization. Due to the birefringence of the laser cavity these two LP modes have slightly different frequencies of operation and are, therefore, referred in the rest of the letter as high and low frequency LP modes.

As it can be observed from Fig. 1(b) the solitary VCSEL emits in its high frequency LP mode at any current within the current operation range, maintaining stable polarization operation. The mode suppression ratio is measured to be equal to $19.5 \mathrm{~dB}$ at $8 \mathrm{~mA}$. However, no special measures, as mentioned in the Introduction, were taken to introduce gain/loss
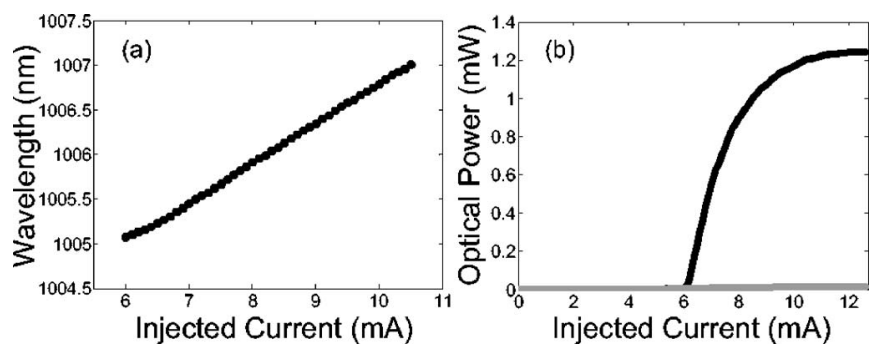

FIG. 1. (a) Wavelength of operation of the solitary VCSEL vs injected current. (b) Polarization resolved PI curve of the solitary VCSEL. Gray and black lines represent the low and the high frequency LP modes, respectively. The measured powers of both modes have been multiplied by the same factor in order to overcome the losses in the detection arm. 


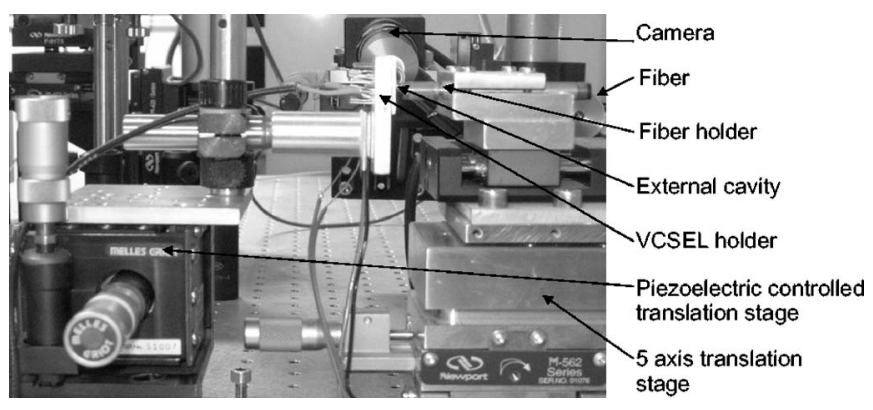

FIG. 2. Picture of the experimental setup.

anisotropies in the VCSEL design. Therefore, on the same wafer one finds either polarization stable VCSELs, like the one presented here, or VCSELs that undergo polarization switching when changing the injected current.

In the experimental setup we create the ESEC by placing an aluminum mirror with a power reflectivity of 0.3 at about $20 \mu \mathrm{m}$ from the top mirror of the VCSEL. In order to do so we glue the mirror on the cleaved facet of a polarization maintaining optical fiber with index matching glue. The fiber is mounted on a five axis translation stage and the VCSEL is mounted on a three axis piezoelectric controlled translation stage which allows precise alignment of the fiber and the VCSEL (see Fig. 2). The external cavity (EC) length is changed by moving the VCSEL along the direction of light emission by means of one of the piezo components of its stage in steps of $20 \mathrm{~nm}$ and with a precision of $2 \mathrm{~nm}$. However, due to the alignment of the charge coupled device camera with respect to system VCSEL-external mirror (see Fig. 2) the absolute value of the EC length is known within $20 \mu \mathrm{m}$ (the resolution of the camera itself is $1 \mu \mathrm{m}$ ). In this way, the EC length reported in the following is somehow relative - the zero corresponds to an EC length smaller than $20 \mu \mathrm{m}$. The polarization resolved optical power is measured at the far end of the polarization maintaining optical fiber as in Ref. 17. Any lateral or angular misalignment decreases the feedback strength as well as the optical power coupled into the fiber. Therefore the proper alignment is achieved by maximizing the optical power measured at the fiber end by using the fine piezomechanical tuning (which keeps the optimal EC length fixed).

Figures 3(a) and 3(b) show the polarization resolved PI curves of the VCSEL obtained with EC lengths equal to 10.05 and $10.52 \mu \mathrm{m}$, respectively. Due to the feedback the VCSEL that in the solitary case showed a stable polarization operation now switches from the high frequency LP mode to

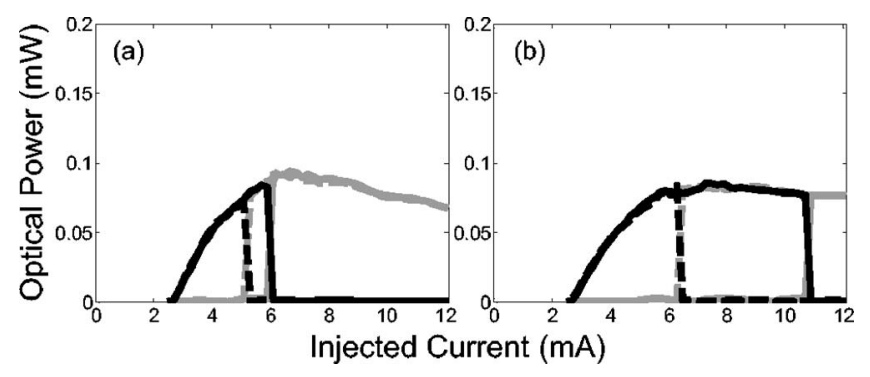

FIG. 3. Polarization resolved PI curve of the VCSEL subject to optical feedback from an ESEC of (a) $10.05 \mu \mathrm{m}$ and (b) $10.52 \mu \mathrm{m}$ in lengths. Gray (black) line represents the low (high) frequency LP mode, while solid (dashed) lines represent the two LP modes for increasing (decreasing) the injection current.

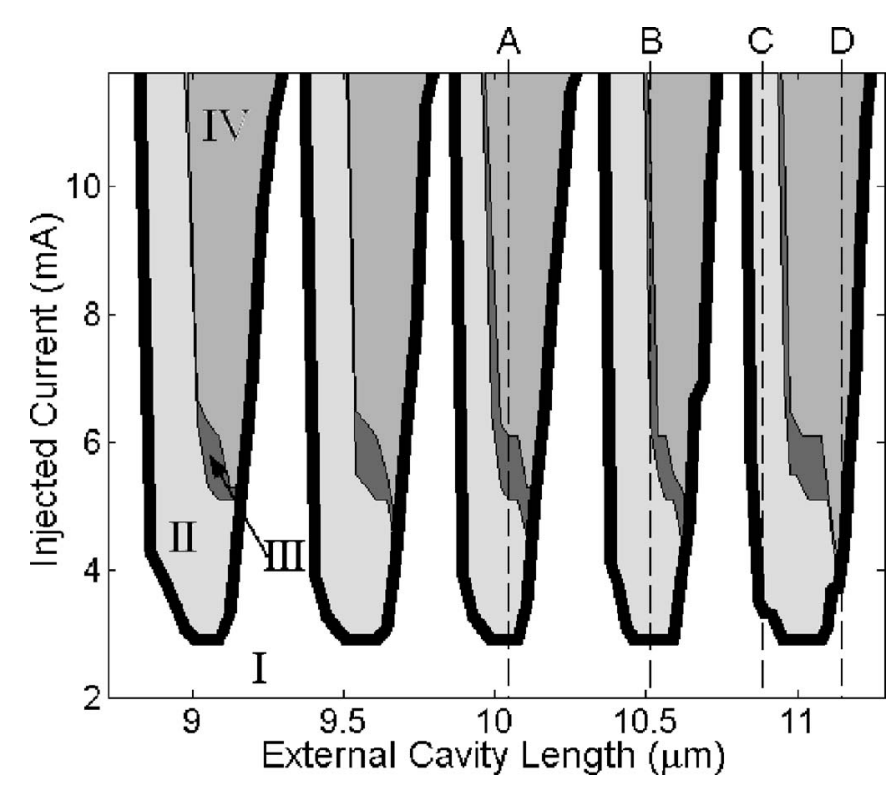

FIG. 4. Experimentally obtained mapping of bistability of a VCSEL subject to isotropic feedback from an ESEC. A gray scale is used to distinguish between different zones. The darkest gray zone (zone III) between zones II and IV represents the bistable region. The thick black line represents the threshold current below which the laser is not emitting (zone I). On the other hand, in zone II (IV) the VCSEL is stable and emits in its high (low) frequency mode.

the orthogonally polarized low frequency LP mode when increasing the injection current. The polarization switching does not occur at the same position when increasing or decreasing the injected current, describing a hysteresis (bistable) region [between 5.2 and $6 \mathrm{~mA}$ in Fig. 3(a)]. Comparing Figs. 3(a) and 3(b) we observe that the currents at which polarization switching takes place are strongly modified [PS currents of 6.4 and $10.8 \mathrm{~mA}$ in Fig. 3(b)].

The impact of the ESEC feedback on the polarization properties of the VCSEL is best illustrated by measuring the polarization resolved PI curve for different EC lengths (we change the EC length in steps of $20 \mathrm{~nm}$ ) and plotting a twodimensional map of the bistable region in the plane injection current-EC length (see Fig. 4). In this way Figs. 3(a) and 3(b) could be extracted as vertical cuts of Fig. 4 at EC lengths equal to $10.05 \mu \mathrm{m}$ (cut A) and $10.52 \mu \mathrm{m}$ (cut B), respectively. From Fig. 4 we observe that the threshold current is strongly modulated with a period equal to half of the VCSEL wavelength. Within each period of modulation and depending on the EC length different polarization resolved PI curves are observed. In some of them we can notice polarization switching from the high frequency mode to the low frequency mode when increasing the injection current (see Fig. 3). We also observe polarization stable PI curves, without any switch of the polarization taking place (see Fig. 5). Such polarization stabilization using ESEC optical feedback has been theoretically predicted in Ref. 16 . We illustrate the polarization stabilization induced by the ESEC feedback in Figs. 5(a) and 5(b), which show the polarization resolved PI curves obtained at EC lengths of 10.89 and $11.15 \mu \mathrm{m}$, respectively (cuts C and D in Fig. 4). In Fig. 5(a) the VCSEL is emitting in its high frequency mode at any injected current (as in the solitary case) while in Fig. 5(b) only the low frequency mode is emitted at any injected current. The mode suppression ratios are measured to be 20 and $18 \mathrm{~dB}$ in Figs. 5(a) and 5(b), respectively, at an injection current of $8 \mathrm{~mA}$. 


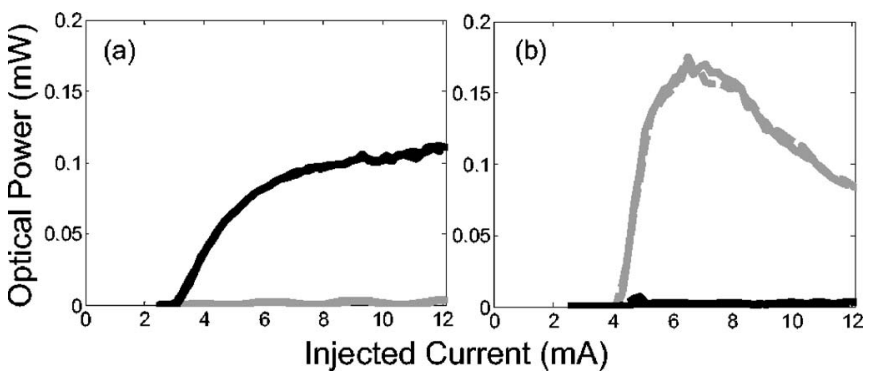

FIG. 5. Polarization resolved PI curve of the VCSEL subject to optical feedback from an ESEC of (a) $10.89 \mu \mathrm{m}$ and (b) $11.15 \mu \mathrm{m}$ in lengths. Gray (black) line represents the low (high) frequency LP mode, while solid (dashed) lines represent the two LP modes for increasing (decreasing) the injection current.

We explain the polarization selection and stabilization in ESEC-VCSEL by the combined effect of the frequency spacing between the two LP modes, the modulation of the mirror losses due to the optical feedback, and the redshift of the emission wavelength with the injection current (see Refs. 16 and 18). The external mirror induces losses in each LP mode that depend on the phase difference between the electric field that reenters the VCSEL after reflecting from the external mirror and the electric field traveling within the VCSEL cavity. Therefore, due to the frequency difference between the LP modes there is a difference in the losses for the two LP modes that is periodically modulated with the EC length. If this modulation is deep enough the VCSEL will emit in the LP mode with smaller external mirror induced looses near the maxima and the minima of modulation, and polarization switching when increasing the injection current will occur near the medium value of the modulation.

In summary, we present experimental evidences showing that isotropic optical feedback from an ESEC can induce switching between the two fundamental transverse modes with orthogonal linear polarization of otherwise polarization stable VCSEL (without optical feedback). The currents at which polarization switching takes place, the width of the bistable region and the threshold currents, are found to be strongly modified when changing the EC length. By properly choosing the EC length within a subwavelength range we can stabilize the polarization of the light emitted by the VCSEL in any of the two fundamental transverse modes of the device (obtained mode suppression ratio larger than $18 \mathrm{~dB}$ ). Therefore, it is possible to utilize ESEC optical feedback to actively control the polarization of the light emitted by a VCSEL at any injected current within the current operation range. Such effect could be used in any application that requires low cost, stable, and eventually fast controllable polarization optical sources, as, for example, optical interconnections, optical fiber sensing, and optical fiber communications.

The authors acknowledge Rainer Michalzik and his coworkers at the Optoelectronics Department of the University of Ulm for providing the VCSELs, and the financial support of the Public University of Navarra, IAP "Photon" network of the Belgian government, FWO-Flanders, GOA and OZR of the VUB, and Project No. TEC-2004-05936-C02-01 of the MEC (Spain).

${ }^{1}$ C. J. Chang-Hasnain, J. P. Harbison, G. Hasnain, A. C. V. Lehmen, L. T. Florez, and N. G. Stoffel, IEEE J. Quantum Electron. 27, 1402 (1991).

${ }^{2}$ A. K. Jansen van Doorn, M. P. van Exter, and J. P. Woerdman, Appl. Phys. Lett. 69, 1041 (1996).

${ }^{3}$ K. D. Choquette, D. A. Richie, and R. E. Leibenguth, Appl. Phys. Lett. 64, 2062 (1994).

${ }^{4}$ B. Ryvkin, K. Panajotov, A. Georgievski, J. Danckaert, M. Peeters, G. Verschaffelt, H. Thienpont, and I. Veretennicoff, J. Opt. Soc. Am. B 16, 2106 (1999)

${ }^{5}$ K. D. Choquette and R. E. Leibenguth, IEEE Photonics Technol. Lett. 6, 40 (1994).

${ }^{6}$ P. Debernardi, J. M. Ostermann, M. Feneberg, C. Jalics, and R. Michalzik, IEEE J. Sel. Top. Quantum Electron. 11, 107 (2005).

${ }^{7}$ H. Uenohara, K. Tateno, T. Kagawa, Y. Ohiso, H. Tsuda, T. Kurokawa, and C. Amano, IEEE Photonics Technol. Lett. 11, 400 (1999).

${ }^{8}$ P. Besnard, M. L. Chares, and G. Stephan, J. Opt. Soc. Am. B 16, 1059 (1999).

${ }^{9}$ M. Sciamanna, K. Panajotov, H. Thienpont, I. Veretennicoff, P. Megret, and M. Blondel, Opt. Lett. 28, 1543 (2003).

${ }^{10}$ T. H. Russell and T. D. Milster, Appl. Phys. Lett. 70, 2520 (1997).

${ }^{11}$ P. Besnard, F. Robert, M. L. Chares, and G. Stephan, Phys. Rev. A 56, 3191 (1997).

${ }^{12}$ Y. Hong, P. S. Spencer, and K. A. Shore, Opt. Lett. 29, 2151 (2004).

${ }^{13}$ C. Masoller and M. S. Torre, IEEE J. Quantum Electron. 41, 483 (2005).

${ }^{14}$ Z. G. Pan, S. Jiang, M. Dagenais, R. A. Morgan, K. Kojima, M. T. Asom, R. E. Leibenguth, G. D. Guth, and M. W. Focht, Appl. Phys. Lett. 63, 2999 (1993).

${ }^{15}$ P. W. Smith, IEEE J. Quantum Electron. 1, 343 (1965).

${ }^{16}$ M. Arizaleta Arteaga, H. J. Unold, J. M. Ostermann, R. Michalzik, H. Thienpont, and K. Panajotov, IEEE J. Quantum Electron. 42, 89 (2006).

${ }^{17}$ M. Arizaleta, H. J. Unold, J. M. Ostermann, R. Michalzik, H. Thienpont, and K. Panajotov, IEEE J. Quantum Electron. 42, 102 (2006).

${ }^{18}$ K. Panajotov, M. Arizaleta, M. Camarena, H. J. Unold, J. M. Ostermann, R. Michalzik, and H. Thienpont, Appl. Phys. Lett. 84, 2763 (2004). 